\title{
La Importancia de la Productividad Científica en la Acreditación Institucional de Universidades Chilenas
}

\author{
Ana M. Barra \\ Facultad de Ciencias Empresariales, Depto de Auditoría y Administración, Universidad del Bío Bío, \\ Avenida Collao 1202. Concepción. Chile (e-mail: abarra@ubiobio.cl)
}

Recibido Jul. 31, 2018; Aceptado Oct. 16, 2018; Versión final Dic. 17, 2018, Publicado Jun. 2019

\begin{abstract}
Resumen
El objetivo de este documento es analizar el impacto de la productividad científica en la acreditación institucional de las universidades que pertenecen al Consejo de Rectores de las Universidades Chilenas. Esto corresponde a 16 universidades estatales y 9 universidades privadas con contribución estatal. La productividad científica se mide considerando los miembros de la facultad que tienen grado de doctorado, el número de trabajos publicados en revistas científicas indexadas en la Web of Science y la cantidad de proyectos Fondecyt (con financiamiento estatal) adjudicados a las instituciones anteriores, factores que influyen notoriamente en la calidad educativa. La metodología es de método mixto a través de técnicas de investigación documental, e informes de ministerios y consorcios. Por otro lado, los criterios estadísticos se definen para cuantificar la relevancia de ciertos factores que afectan a la acreditación institucional. Se concluye que existe una relación lineal positiva entre los años de acreditación institucional y el número de miembros de la facultad que poseen un doctorado, trabajos de investigación publicados y proyectos.
\end{abstract} las publicaciones

\section{The Importance of Scientific Productivity in the Institutional Accreditation of Chilean Universities}

\begin{abstract}
The aim of this paper is to analyze the impact of scientific productivity on the institutional accreditation of universities that belongs to the Council of Rectors of Chilean Universities. These council is formed by 16 state universities and 9 private universities with state contribution. Scientific productivity is measured considering the faculty members that hold a PhD degree, the number of papers published in scientific journals indexed in the Web of Science, and the amount of Fondecyt projects (with state support) awarded to these institutions, factors that notoriously influence teaching quality. The methodology is of the mixed-type method through techniques of documentary research, and reports from ministries and consortiums. On the other hand, statistical criteria are defined to quantify the importance of the factors that affect institutional accreditation of these institutions. It is concluded that there is a positive linear relationship between the years of institutional accreditation and the number of faculty members holding $\mathrm{PhD}$ degree, the number of publications and the number of projects.
\end{abstract}

Keywords: accreditation; scientific productivity; higher education; university education; quality of papers 


\section{INTRODUCCIÓN}

La mayoría de los países latinoamericanos instaló sus sistemas de aseguramiento de la calidad en la segunda mitad de la década de los 90, a excepción de unos pocos países que ya tenían algún tipo de sistema de regulación o control instalado con anterioridad (Brasil, Chile, Colombia, Costa Rica, Cuba, México y República Dominicana) (Perez, 2018). Los retos de la educación superior para el Siglo XXI plantean la necesidad de un nuevo proceso educativo, fundamentado en los principios de excelencia, calidad y pertinencia. Calidad que se sustenta en la formación y superación de los recursos humanos. Es así como la excelencia académica constituye uno de los elementos de mayor importancia y controversia en la educación superior contemporánea; pues su determinación se encuentra estrechamente vinculada a los procesos de la evaluación curricular y la acreditación académica de las Universidades (Salas, 2000). La mayoría de los sistemas de Evaluación y Acreditación de Educación Superior, tienen como finalidad el mejoramiento o promoción de la calidad de la educación superior y la acreditación de las carreras o programas que cumplan con estándares o criterios previamente definidos, en combinación con el respectivo proyecto institucional. Los procedimientos son bastante similares, e incluyen la autoevaluación realizada por la propia institución, la visita a la institución realizada por pares evaluadores externos y la evaluación final (Perez, M, 2018). Es un proceso voluntario al que se someten las instituciones de Educación Superior autónomas del país, así como las carreras de pregrado, programas de postgrado y especialidades del área de la salud que ellas imparten, para contar con una certificación de calidad de sus procesos internos y sus resultados.

Según la Comisión Nacional de Acreditación Institucional (CNA), las universidades, institutos profesionales y centros de formación técnica autónomos pueden someterse voluntariamente a acreditación institucional, proceso cuyo objeto es evaluar el cumplimiento de su proyecto corporativo y verificar la existencia de mecanismos eficaces de autorregulación y de aseguramiento de la calidad, así como propender al fortalecimiento de su capacidad de autorregulación y al mejoramiento continuo. Metodológicamente, el proyecto piloto de acreditación institucional fijó áreas en las que se desarrolla la evaluación de los mecanismos de aseguramiento de la calidad. Estableció como áreas mínimas de evaluación la gestión institucional y la docencia de pregrado, puesto que son esenciales para toda institución de educación superior. Además de las mínimas ya mencionadas, y con el fin de dar cuenta de la diversidad del sistema de educación superior, las instituciones pueden evaluarse en áreas adicionales, entre las que se cuentan: la investigación, la docencia de posgrado, la vinculación con el medio y la gestión de su infraestructura y equipamiento (Zapata, 2018). En el caso de las carreras de Medicina y Pedagogías, la acreditación es obligatoria, según lo establece la Ley 20.129. Hoy en día cobra vital importancia el conjunto de procesos de investigación formativa como medio de formación de aquellos actores sociales que generarán en un futuro, conocimiento. La investigación formativa se refiere a la formación en y para la investigación a través de actividades propias de la investigación, pero que no necesariamente están involucradas en proyectos que pretenden lograr resultados científicos. Las universidades han buscado permanentemente mecanismos que le permitan evaluar su calidad, considerándose este uno de los temas importantes en la definición de una política universitaria para progresar en el futuro (Embid-Michavilla, 2001). En este sentido, un mecanismo que ha alcanzado relevancia en la medición de la calidad de las Instituciones de Educación Superior, Carreras o Programas de Pregrado, y Programas de Postgrado, se asocia al logro de una certificación externa, la "acreditación".

El proceso de acreditación institucional es complejo y requiere de varias etapas a realizar para finalizar con la acreditación institucional obtenida. Ante esto, para las universidades, un indicador de éxito del proceso de acreditación se asocia normalmente a la cantidad de años de acreditación obtenidos, y a las áreas opcionales que se logran acreditar. Sin embargo, existen otras maneras de evidenciar que existen otras variables institucionales que determinan, además la acreditación institucional. Como son: el número de áreas acreditadas, el número de docentes full time, número de docentes con posgrado, número de docentes jornada completa con grado de doctor, número de proyectos FONDECYT adjudicados los últimos tres años, número de programas de doctorados acreditados, número de programas de magíster acreditados, número de volúmenes (textos por alumno), número de laboratorios, entre otros (Cancino y Schmal 2014)

\section{Acreditación Institucional}

Respecto a la investigación desarrollada, la CNA plantea ciertas orientaciones relacionadas con este proceso, "consistente con la Ley $\mathrm{N}^{\circ} 20.129$, la acreditación institucional tiene por principal orientación la evaluación de los mecanismos de autorregulación de la calidad de las instituciones y sus resultados". Se trata de una orientación particular, puesto que enfatiza que la calidad es esencialmente responsabilidad de las propias instituciones y no de un organismo externo a ellas, sin perjuicio de que la garantía pública de calidad sea provista externamente mediante procesos sistemáticos de evaluación.La acreditación institucional está planteada como una auditoría académica, esto es, la evaluación de la capacidad institucional para la autorregulación, entendida como el conjunto de políticas, mecanismos, procedimientos y acciones destinados a determinar si la institución está efectivamente avanzando hacia el logro de sus propósitos y objetivos (Zapata, 2018). 
El proceso de acreditación institucional definido por la CNA Chile, parte del supuesto de que las instituciones de educación superior que quieren acreditarse cuentan con políticas y mecanismos eficaces de autorregulación, esto es, que tienen propósitos explícitos, que verdaderamente aspiran a lograr; que estos propósitos satisfacen los requerimientos básicos propios de una institución de educación superior; que organizan sus actividades de manera de avanzar eficaz y eficientemente hacia el logro de sus propósitos; que verifican periódicamente su grado de avance sobre el particular; por último, que ajustan sus acciones de acuerdo a los resultados de esa verificación. Por consiguiente, el principal objetivo es "la evaluación, promoción y garantía pública de los procesos de autorregulación de las instituciones de educación superior en áreas determinadas, para lo cual se requiere evaluar la existencia formal, la aplicación sistemática y los resultados de las políticas y mecanismos que apuntan al cumplimiento de los fines de una institución, garantizando así su calidad." (Guía para la Autoevaluación Interna Acreditación Institucional Universidades CNA-Chile, 2016).

\section{La evaluación interna}

El proceso se inicia con una autoevaluación, referida al análisis de las políticas y mecanismos institucionales destinados a asegurar la calidad de la gestión institucional y la docencia de pregrado, así como de cualquier área adicional que la institución haya decidido incorporar. Igualmente debe contemplar el análisis de los resultados de la aplicación de dichas políticas y mecanismos. (CNA-Chile, 2016). La clave del proceso de evaluación interna es la identificación y posterior análisis de la aplicación de los mecanismos de aseguramiento de la calidad al interior de la institución y en sus diversos niveles (Zapata, G, 2018).

\section{Áreas de evaluación}

El proceso de acreditación considera cinco áreas de evaluación: dos mínimas y tres adicionales (CNA-Chile, 2016, pp.12 -18 y Reglamento sobre Áreas de Acreditación, Resolución Exenta № 01, de 5 de febrero de 2013 de CNA):

\section{Gestión institucional (mínima, obligatoria).}

Se entiende el conjunto de políticas y mecanismos destinados a organizar las acciones y recursos -materiales, humanos y financieros- de la institución, en función de sus propósitos y fines declarados. Considera la organización y estructura institucional, el sistema de gobierno y la administración de recursos humanos, materiales y financieros.

\section{Docencia de pregrado (mínima, obligatoria).}

En este ámbito se considera el conjunto de políticas y mecanismos institucionales destinados a asegurar la calidad de la formación de pregrado, con especial énfasis en los aspectos relacionados con el diseño y aprobación de los programas ofrecidos; con su implementación y seguimiento; y con un análisis de sus resultados y los mecanismos para revisar y modificar el currículo, la organización de los programas, los métodos pedagógicos, los recursos humanos y materiales asignados a los programas o cualquier otro aspecto que afecte la calidad de la formación entregada.

\section{Docencia de postgrado}

Las Instituciones que opten por esta área deben considerar todas las actividades de posgrado que realice la Universidad. En caso de que sólo cumplan las condiciones establecidas para los programas de doctorado 0 maestría, CNA Chile determinará si la institución es susceptible de ser evaluada en el área.

4. Investigación

Por investigación se entienden las actividades sistemáticas de búsqueda de nuevo conocimiento, que impactan sustantivamente en la disciplina, tema o área a la que pertenecen. Sus resultados se expresan en publicaciones o en patentes. Para optar por esta área, la institución debe desarrollar actividades sistemáticas de investigación de alto nivel en cuanto a la rigurosidad de los proyectos y a su contribución al desarrollo disciplinario o científico, en diversas áreas de su quehacer, expresadas en un conjunto significativo de proyectos de investigación integrados al sistema nacional de ciencia y tecnología. La evaluación para la acreditación refiere a las políticas y mecanismos institucionales destinados a asegurar la calidad de la investigación.

\section{Vinculación con el medio}

La vinculación con el medio se refiere al conjunto de nexos establecidos con el medio disciplinario, artístico, tecnológico, productivo o profesional, con el fin de mejorar el desempeño de las funciones institucionales, de 
facilitar el desarrollo académico y profesional de los miembros de la institución y su actualización o perfeccionamiento, o de cumplir con los objetivos institucionales. Para optar por esta área, las instituciones deben contar con mecanismos sistemáticos de vinculación con el medio, que refieran a la parte sustantiva del quehacer de la institución y que tengan un impacto significativo en su área de influencia. La evaluación para la acreditación se refiere a las políticas y mecanismos institucionales destinados a asegurar la calidad de esta actividad.

\section{Productividad Científica}

Según Colciencias (2016), la investigación científica corresponde a "la investigación y el desarrollo experimental, que comprenden el trabajo creativo llevado a cabo de forma sistemática para incrementar el volumen de conocimientos, incluido el conocimiento del hombre, la cultura y la sociedad, y el uso de esos conocimientos para crear nuevas aplicaciones." Además, menciona que uno de los principales objetivos de la actividad científica es hacer accesible y útil la investigación, permitiendo con ello la circulación de conocimiento. El impacto de una publicación científica, entendida como la medida de su relevancia e influencia mediante el número de citaciones, es lo que refleja hasta qué punto dicho objetivo se ha alcanzado.

Además, se reconoce que el estudio de la productividad científica, tradicionalmente expresada en la cantidad de publicaciones por investigador, es uno de los indicadores más usado para medir el desempeño del sistema científico en la producción de conocimiento (De Filippo y Fernández, 2002).

El término Investigación y desarrollo experimental engloba tres actividades: investigación básica, investigación aplicada y desarrollo experimental:

\section{1) Investigación básica.}

Consiste en trabajos experimentales o teóricos que se emprenden principalmente para obtener nuevos conocimientos acerca de los fundamentos de los fenómenos y hechos observables, sin pensar en darles ninguna aplicación o utilización determinada". Independientemente del área del conocimiento.

\section{2) Investigación aplicada.}

Consiste también en trabajos originales realizados para adquirir nuevos conocimientos; sin embargo, está dirigida fundamentalmente hacia un objetivo práctico específico", independientemente del área del conocimiento. La investigación aplicada se emprende para determinar los posibles usos de los resultados de la investigación básica, o para determinar nuevos métodos o formas de alcanzar objetivos específicos predeterminados.

\section{3) Desarrollo experimental.}

Consiste en trabajos sistemáticos que aprovechan los conocimientos existentes obtenidos de la investigación y/o la experiencia práctica, y está dirigido a la producción de nuevos materiales, productos o dispositivos; a la puesta en marcha de nuevos procesos, sistemas y servicios, o a la mejora sustancial de los ya existentes. Según formato de publicaciones WOS, la información que se pone a disposición en esta ocasión, tiene como objetivo principal reconocer a simple vista la relevancia que cobran las publicaciones científicas según su tipo de formato o soporte.

Según Salazar (2008), la producción científica hoy en día, es un tema de análisis permanente de cara a abordar con asertividad la calidad de la educación. Esta área, en Chile es fundamentalmente desarrollada por las universidades, que en este sentido tienen como propósito final contribuir a transformar al país en una sociedad basada en el conocimiento, cumpliendo así un importante rol público. Por tal motivo, el Estado dota de recursos económicos a las universidades con el fin que puedan realizar su actividad investigadora y, por lo tanto, estas deben responder según las exigencias y directrices que satisfagan lo requerido (Díaz, 2013). Esto reviste mayor importancia en Latinoamérica, donde recién se están instalando procesos de evaluación docente, a diferencia de muchos países desarrollados, que exigen que los profesores que laboran en el sector público acrediten sus competencias cada 4 o 5 años. Por otro lado, para Valera y De la Gala (2001), la productividad científica es un indicador de la actividad científica que permite determinar el crecimiento de la ciencia a través del número de trabajos publicados, su evolución cronológica, la productividad de los autores, instituciones o regiones, así como un análisis del grado de colaboración entre científicos o instituciones, y la estructura y dinámica de los colectivos de investigadores.

En relación a las variables para medir la productividad científica, éstas no están normadas por las instituciones, sin embargo, la cantidad de publicaciones en revistas indexadas y proyectos de transferencia tecnológica con impacto para la región o país, parecieran ser los tópicos que más se repiten a la hora de 
abordar temas de productividad en las universidades. Es así como Buela-Casal et al. (2010), mencionan las variables de: artículos en revistas indexadas, tramos de investigación, proyectos de I+D, tesis doctorales, becas obtenidas, doctorados con mención en calidad y patentes.

Y finalmente, también hay que considerar los períodos o ciclos de vida en la cual se encuentran las instituciones de educación superior; al respecto, y como lo señalan Espinoza y González (2009), el primer período, es el fundacional y se sustenta en el esfuerzo personal de investigadores que diseñan e implementan programas avanzados para un grupo muy selecto de estudiantes, con el apoyo de las redes internacionales que han generado como fruto de su trabajo de investigación. El segundo período se caracteriza por la creación del Fondo Nacional de Desarrollo Científico y Tecnológico, órgano que apoya con fondos concursables, la investigación y el desarrollo de estudios de posgrado. Y finalmente, desde 1999 se fortalecen infraestructuras, creación de nuevos programas y universidades que ofrecen doctorados y se potencian los magísteres. Aun cuando el crecimiento de la producción científica, así como su recopilación ha impulsado, en estas últimas décadas, el uso de la bibliometría y la producción correlativa de indicadores para evaluar los resultados de la misma, cabe señalar que su alcance ha sido y continúa siendo un punto de debate dentro de la comunidad científica (Oyarzun, 2007), discutiéndose sus orígenes, metodologías y límites técnicos (Vargas, 2007).

\section{Formación Académica}

La unión entre innovación y educación es un binomio inseparable dentro de la Formación Profesional sobre todo cuando se persigue una formación de calidad y actualizada con las nuevas tecnologías. La Formación Profesional es innovadora y se basa en tres pilares: 1) Innovación tecnológica; 2) Innovación pedagógica; Y 3) Colaboración con las empresas.

Es así como los programas de formación docente, se configuran como el mecanismo catalizador de la educación en la sociedad, trayendo consigo gran responsabilidad, ya que son los encargados de la preparación de los académicos, quienes se caracterizan por tener un conocimiento amplio, una sólida capacidad pedagógica y la disposición para guiar y sostener a los estudiantes, además de una comprensión del clima cultural y social de la educación (Szilagyi y Szecsi, 2011). La formación profesional es un ámbito de la educación que está en permanente evolución, porque tiene que integrar las nuevas vías de especialización que van surgiendo, así como adaptarse a las necesidades cambiantes del mercado laboral y, sobre todo, porque ha de mejorar para ofrecer la formación más competitiva posible. Y la forma más adecuada de llevar a cabo esta tarea es, hoy en día, integrar dentro del proceso el concepto de innovación en toda su magnitud.

La Comisión Técnica del Consejo Nacional Educación Trabajo (2001), indica que la Formación Profesional requiere, cada vez más, de procesos educativos integrales, integradores y permanentes, orientados hacia una polivalencia tecnológica y hacia una rápida adaptación a contextos técnico-profesionales diversos. La innovación, desde el punto de vista de la formación profesional (FP), es la capacidad de proponer modificaciones o nuevas soluciones con el fin de mejorar los resultados. Desde el punto de vista empresarial, innovar en la FP supone transformar el conocimiento y las ideas en servicios que se inserten en el mercado dando respuesta a una demanda existente. La formación profesional es una herramienta que favorece que los ciudadanos mejoren sus posibilidades de proyección profesional y personal y que las empresas aumenten su competitividad.

\section{METODOLOGÍA}

En esta sección se da a conocer la muestra utilizada y el o los instrumentos de medida comprendidos en este estudio.

\section{Descripción de la muestra}

El universo de estudio comprende a las universidades pertenecientes al Consejo de Rectores de las Universidades Chilenas. La población total sobre la cual se aplica esta investigación es de 25 universidades (16 universidades estatales y 9 tradicionales privadas con aporte estatal) que representan el $100 \%$ del total de las universidades del $\mathrm{CRUCH}$.

\section{Instrumento de medida}

El instrumento de medida utilizado para este trabajo es una caracterización descriptiva del proceso de acreditación de las universidades del CRUCH. Para ello, se estructuró un instrumento con cuatro dimensiones: dimensión 1: acreditación de Posgrado en la universidad; dimensión 2: número de doctores; dimensión 3: número de publicaciones y dimensión 4: número de proyectos Fondecyt. Esto se fundamenta debido a que estas dimensiones son las más influyentes en la productividad científica de los académicos y de la universidad 
en sí, siendo relevante estudiarlas y analizarlas para identificar el grado de influencia de cada una de ellas. La explicación de estas dimensiones se encuentra en la tabla 1.

\section{RESULTADOS}

Para verificar el criterio de la normalidad, se realiza una prueba de hipótesis de normalidad para cada caso, para ver si la variable se distribuye normalmente. Los resultados de la prueba de normalidad de Shapiro-Wilk son presentados en la tabla 2. En todos los casos a excepción de la variable años de acreditación institucional, el valor - p es significativo (menor a 0,05), es decir, hay evidencia para indicar que estas variables no se distribuyen bajo la distribución normal.

Tabla 1: Explicación de las dimensiones

\begin{tabular}{|c|c|}
\hline Dimensión & Explicación de las variables \\
\hline Acreditación en Posgrado & $\begin{array}{l}\text { La acreditación de programas de posgrado correspondientes a magíster, } \\
\text { doctorado y especialidades en el área de la salud y de otros niveles equivalentes } \\
\text { tiene por objeto certificar la calidad de los programas ofrecidos por las } \\
\text { instituciones autónomas de educación superior, en función de los propósitos } \\
\text { declarados por la institución que los imparta y los criterios o estándares } \\
\text { establecidos para este fin por la comunidad científica o disciplinaria } \\
\text { correspondiente. Esta acreditación será voluntaria mientras la legislación } \\
\text { vigente no disponga otra cosa. }\end{array}$ \\
\hline Número de doctores & $\begin{array}{l}\text { Los programas de doctorado corresponden al más alto grado otorgado por una } \\
\text { o más universidades. Comprende un proceso sistemático de investigación que } \\
\text { culmina con la elaboración, defensa y aprobación de una tesis. Deben estar } \\
\text { sustentados por académicos con líneas de investigación activas y productividad } \\
\text { acorde a las mismas. }\end{array}$ \\
\hline Número de publicaciones & $\begin{array}{l}\text { La investigación científica corresponde a la investigación y el desarrollo } \\
\text { experimental, que comprenden el trabajo creativo llevado a cabo de forma } \\
\text { sistemática para incrementar el volumen de conocimientos, incluido el } \\
\text { conocimiento del hombre, la cultura y la sociedad, y el uso de esos } \\
\text { conocimientos para crear nuevas aplicaciones. }\end{array}$ \\
\hline Número de proyectos Fondecyt. & $\begin{array}{l}\text { El Fondo Nacional de Desarrollo Científico y Tecnológico, Fondecyt, tiene por } \\
\text { objetivo estimular y promover el desarrollo de investigación científica y } \\
\text { tecnológica básica, y es el principal fondo de este tipo en el país. Creado en } \\
1981 \text {, ha financiado más de } 16 \text { mil proyectos de investigación cuyos impactos } \\
\text { han beneficiado tanto a la comunidad científica como a la sociedad en general. }\end{array}$ \\
\hline
\end{tabular}

Tabla 2: Prueba de Shapiro-Wilk

\begin{tabular}{|l|r|r|r|}
\hline \multicolumn{1}{|c|}{ Variables } & \multicolumn{1}{c|}{ Estadístico } & \multicolumn{1}{c|}{ g. I. } & \multicolumn{1}{c|}{ Sig. } \\
\hline Año de acreditación institucional & 0.920 & 25 & 0.052 \\
\hline № de doctores & 0.836 & 25 & 0.001 \\
\hline № de publicaciones & 0.634 & 25 & 0.000 \\
\hline № de proyectos Fondecyt. & 0.606 & 25 & 0.000 \\
\hline
\end{tabular}

\section{Descriptivos}

Los intervalos de confianza ofrecen una manera de estimar, con alta probabilidad 99\%, un rango de valores en el que se encuentra el valor de una determinada variable. Bajo la hipótesis de normalidad (Tabla 3). Es decir, los años de acreditación institucional de una institución estatal estarán entre $(3,95 ; 5,58)$ con un 99\% de confianza; y en una institución privada con aporte estatal los años de acreditación estarán entre $(4,18$; 6,82 ) con un $99 \%$ de confianza. La tabla 3 resume estos resultados. Si se busca conocer la relación entre dos o más variables, el no cumplimiento del supuesto de normalidad conlleva la necesidad de recurrir a pruebas estadísticas no paramétricas. La tabla 4 describe las variables que se utilizarán. 
Tabla 3: Intervalo de confianza para la media al $99 \%$

\begin{tabular}{|l|l|l|}
\hline & Tipo de institución & \multicolumn{1}{|l|}{ Intervalo de confianza para la media al 99\% } \\
\hline \multirow{3}{*}{ Años de acreditación institucional } & \multirow{2}{*}{ Estatal } & Límite inferior: 3,95 \\
\cline { 2 - 3 } & \multirow{2}{*}{ Privada con aporte estatal } & Límite superior: 5,58 \\
\cline { 3 - 3 } & & Límite inferior: 4,18 \\
\hline
\end{tabular}

\section{Método no paramétrico}

En el caso de las variables cualitativas dicotómicas como "tipo de institución" y "acreditación en posgrado", no es correcto utilizar la prueba de Kruskal-Wallis ya que solo poseen dos grupos o categorías. Por lo cual la prueba adecuada para estas variables es la prueba $U$ de Mann-Whitney la cual procede en forma similar a la anterior, pero en esta prueba si es posible trabajar con variables dicotómicas. Hipótesis para la variable de agrupación número de doctores: H0: No existe diferencia en la mediana del número de años de acreditación Institucional respecto al número de doctores en dicha institución; H1: El número de doctores tiene relación con el número de años de acreditación Institucional

Al analizar el resultado (tabla 4), se observa que en la variable "tipo de institución" el valor-p es mayor a 0.05 , entonces, no existe suficiente evidencia para rechazar $\mathrm{H}_{0}$, por lo cual se sigue asumiendo $\mathrm{H}_{0}$ verdadero. Por lo tanto, podemos decir que la variable tipo de institución es independiente del número de años de acreditación institucional. Sin embargo, en todas las variables restantes se observa que si existe relación entre estas variables y el número de años de acreditación Institucional.

Tabla 4: Pruebas no paramétricas

\begin{tabular}{|l|l|r|r|}
\hline Variable & Prueba no paramétrica & Estadístico & Valor- $p$ \\
\hline Tipo de Institución & U de Mann-Whitney & 42,500 &, 125 \\
\hline Acreditación en Posgrado & U de Mann-Whitney & 7,500 &, 000 \\
\hline Número de doctores & Kruskal-Wallis & 12,172 &, 016 \\
\hline Número de publicaciones & Kruskal-Wallis & 15,094 &, 002 \\
\hline Número de proyectos & Kruskal-Wallis & 12,842 &, 002 \\
\hline
\end{tabular}

Los resultados de las pruebas no paramétricas indican que el número de años de acreditación institucional es independiente del tipo de institución (Valor-p $=0,125)$. Conjuntamente, podemos decir que el número de años de acreditación institucional tiene relación con los diferentes niveles de las variables: Acreditación en Posgrado (valor- $\mathrm{p}=0.000$ ), № de doctores (valor- $\mathrm{p}=0.016$ ), № de publicaciones (valor $-\mathrm{p}=0.002$ ) y № de proyectos Fondecyt (valor- $\mathrm{p}=0.002$ ).

\section{Análisis de regresión}

Es importante destacar que el Análisis de Regresión no establece relaciones causales entre las variables, sólo establece asociaciones entre ellas. Las variables involucradas son las siguientes: Y: Años de acreditación Institucional, $\mathrm{X}_{1}$ : Acreditación en Posgrado, y $\mathrm{X}_{2}$ : Número de publicaciones en web of Science durante el año 2015. Se postula el siguiente modelo que representa la relación entre las observaciones de $\left(X_{1}, X_{2}\right)$. Para medir la bondad del ajuste se utiliza el $\mathrm{R}^{2}$ y $\mathrm{R}^{2}$ corregido (tabla 5), por lo cual podemos decir que las variables expresadas en el modelo de regresión que se presenta a continuación explican el $75,5 \%$ de la variabilidad del número de años de acreditación institucional. Por otra parte, se observa que el $\mathrm{R}^{2}$ corregido es menor al $\mathrm{R}^{2}$, tal comportamiento es esperado.

Tabla 5: Resumen del modelob

\begin{tabular}{|c|c|c|c|c|c|}
\hline Modelo & $R$ & $R$ cuadrado & $\begin{array}{c}\text { R cuadrado } \\
\text { corregida }\end{array}$ & $\begin{array}{c}\text { Error típ. De la } \\
\text { estimación }\end{array}$ & $\begin{array}{c}\text { Durbin- } \\
\text { Watson }\end{array}$ \\
\hline 1 &, $869^{a}$ &, 755 &, 732 &, 597 & 2,470 \\
\hline
\end{tabular}


En la tabla 6 se observa la información sobre los coeficientes del modelo. El resultado se lee de la siguiente forma:

$$
\begin{aligned}
& \ddot{Y}=\beta 0+\beta 1 \times X 1+\beta 2 \times X 2+\varepsilon \\
& \ddot{Y}=6,187-1,212 \times X 1+0,451 \times X 2+\varepsilon
\end{aligned}
$$

El término constante vale $\beta \_0=6,187$; y se puede interpretar como el número de años de acreditación institucional en el caso hipotético de que las demás variables predictoras expresaran un valor cero (ausencia de respuesta) en forma simultánea para la misma institución. Además, de la tabla se contrasta la hipótesis de coeficientes de regresión. Donde:

$$
\begin{aligned}
& \mathrm{H} 0: \text { El coeficiente } \beta \_i=0 v s \\
& \mathrm{H} 1: \beta_{-} i \neq 0, \forall \mathrm{i}
\end{aligned}
$$

\begin{tabular}{|c|c|c|c|c|c|c|}
\hline & \multirow{2}{*}{ Modelo } & \multicolumn{2}{|c|}{ Coeficientes no estandarizados } & \multirow{2}{*}{$\begin{array}{c}\text { Coeficientes tipificados } \\
\text { Beta }\end{array}$} & \multirow{2}{*}{$\mathrm{T}$} & \multirow{2}{*}{ Sig. } \\
\hline & & $\mathrm{B}$ & Error típ. & & & \\
\hline \multirow{3}{*}{1} & (Constante) & 6,187 & ,598 & & 10,350 & ,000 \\
\hline & Acreditación en Posgrado & $-1,212$ & ,290 &,- 532 & $-4,182$ & 000 \\
\hline & Número de publicaciones & ,451 & ,127 & ,452 & 3,555 & ,002 \\
\hline
\end{tabular}

Se observa que todos los coeficientes para las variables indicadoras son significativos ( $P$-valor $<<0)$, es decir, se rechaza la hipótesis nula.

Tabla 6: Coeficientes ${ }^{a}$

\section{Correlación}

Por otro lado, al realizar una correlación, los resultados indican que existe una relación lineal positiva entre los años de acreditación institucional y el número de doctores, el número de publicaciones y el número de proyectos Fondecyt, es decir, son directamente proporcionales. Además, se observa que los años de acreditación institucional son inversamente proporcionales a la acreditación en posgrado. Y no tiene una relación lineal importante con el tipo de institución. La Tabla 7 muestra estos resultados.

Tabla 7: Correlación

\begin{tabular}{|c|c|c|c|c|c|}
\hline \multicolumn{7}{|c|}{ Correlación de Pearson } \\
\hline & $\begin{array}{c}\text { Tipo de } \\
\text { Institución }\end{array}$ & $\begin{array}{c}\text { Acreditación } \\
\text { en Postgrado }\end{array}$ & $\begin{array}{c}\text { No de } \\
\text { doctores }\end{array}$ & $\begin{array}{c}\text { No de publicaciones } \\
\text { (Web of Science) }\end{array}$ & $\begin{array}{c}\text { No de proyectos } \\
\text { Fondecyt. 2017 }\end{array}$ \\
\hline $\begin{array}{c}\text { Años de acreditación } \\
\text { Institucional }\end{array}$ &, 303 &,- 783 &, 766 &, 764 &, 744 \\
\hline
\end{tabular}

Simultáneamente, el análisis de regresión concluye que las variables X1 y X2 presentan una relación estadísticamente significativa en el uso de estimar el número de años de acreditación institucional, bajo la siguiente expresión lineal:

$$
\mathrm{Y}=6,187-1,212 \times \mathrm{X}_{1}+0,451 \times \mathrm{X}_{2}
$$

\section{DISCUSION}

En primer lugar, se menciona una pequeña brecha entre las instituciones de carácter estatal y privada, considerando que los recursos invertidos en cada una de ella son notoriamente distintos, obteniendo resultados respecto a los años de acreditación institucional de una institución estatal estarán entre $(3,95 ; 5,58)$ con un $99 \%$ de confianza. Y en una institución privada con aporte estatal los años de acreditación estarán entre $(4,18 ; 6,82)$ con un $99 \%$ de confianza. 
Finalmente, un análisis de correlación realizada, se observa que los años de acreditación institucional son inversamente proporcionales a la acreditación en posgrado, y no tiene una relación lineal importante con el tipo de institución. Esto último tiene lógica, pues si bien el área de posgrado es un ámbito relevante dentro de lo que conlleva a la productividad científica, pero ésta área no es obligación para acreditar una institución, las áreas mínimas de evaluación son Gestión Institucional y Docencia de Pregrado, las 3 restantes, Docencia de Posgrado, Investigación y Vinculación con el Medio, son áreas adicionales a evaluar, por lo que esto puede explicar la relación inversa que se manifiesta.

A lo anterior se suma que las universidades actuales se encuentran frente al desafío de insertarse en un mundo complejo, con nuevas exigencias de profesionalismo y competencias emergentes asociadas a la sociedad del conocimiento (Espinoza y González, 2012), por tanto, los desafíos en cuanto a instaurar y mejorar los procesos de aseguramiento de la calidad son tremendos, al igual que la inversión que deben hacer las universidades en todos aquellos aspectos que contribuyen a ello.

\section{CONCLUSIONES}

De acuerdo a los resultados y de su discusión y análisis, se pueden extraer las siguientes principales conclusiones:

1) Existe una relación lineal positiva entre los años de acreditación institucional y el número de doctores, el número de publicaciones y el número de proyectos Fondecyt, es decir, son directamente proporcionales; 2 ) Se observa que los años de acreditación institucional son inversamente proporcionales a la acreditación en posgrado, y no tiene una relación lineal importante con el tipo de institución que se estudie; y 3) La variable de productividad científica, presenta una relación estadísticamente significativa en relación al número de años de acreditación institucional. 4) Existe sustento para indicar que la productividad científica, resulta importante para la acreditación institucional.

Las revistas científicas, junto con las pautas y reglas que regulan su funcionamiento, son el canal por el cual los investigadores hacen público de manera "oficial" el resultado de su trabajo. El conjunto de las publicaciones científicas encarna, entonces, el valor del conocimiento disponible y, a su vez, demarca el campo y da escenario a los debates científicos (Barrere et al., 2008)

Todo lo expuesto, manifiesta la relevancia de la productividad científica para lograr el objetivo de cada institución, que es obtener la acreditación institucional, donde se verifica la calidad procesos internos y resultados, manifestando consistencia en cada una de las áreas evaluadas, entendiendo además que las universidades se consideran como un espacio que reúne a investigadores, estudiantes y docentes, que deberían participar y fomentar activamente el desarrollo intelectual-científico, mediante el progresivo aumento del número de publicaciones en revistas indexadas y de realización de artículos científicos en sí, consolidando una materia relevante y que resulta vital, para concretar la acreditación universitaria.

\section{REFERENCIAS}

Buela-Casal, G., M. Bermúdez y otros cuatro autores, Relación de la productividad y eficiencia en investigación con la financiación de las comunidades autónomas españolas, ISSN: 0214-9915, Psicothema, 22(4), 924-931 (2010)

Barrere, R., M. Bageneta y L. Mata, Sistemas científicos complejos y su abordaje metodológico. Centro Argentino de Información Científica y Tecnológica (CAICYT-CONICET), 335-376 (2008)

Cancino, V. y R. Schmall, Sistema de Acreditación Universitaria en Chile: ¿Cuánto hemos avanzado?, doi:10.4067/S071807052014000100003, Estudios Pedagógicos 11(1), 41-60 (2014)

Colcencias, Política Nacional para mejorar el impacto de las publicaciones científicas nacionales. Departamento administrativo de ciencia, tecnología e innovación- Colciencias, Dirección de Fomento a la Investigación (2016)

Colombia Científica, Definiciones y Conceptos (2017)

Comisión Nacional de Acreditación (2017)

Comisión Nacional de Acreditación, Acreditación de programas de postgrado (2017)

Comisión Nacional de Acreditación. Criterios vigentes para la acreditación de postgrados (2016)

Comisión Técnica del Consejo Nacional Educación Trabajo, Materiales de Trabajo para la Formulación de un Acuerdo Marco sobre Formación Profesional, 2-16 (2001)

De Filippo, D. y M. Fernández, Bibliometría: Importancia de los indicadores bibliométricos. En: El Estado de la Ciencia. Principales Indicadores de Ciencia y Tecnología. Iberoamericanos / Interamericanos, Capítulo 2.8. Ed. RICYT / CYTED / REDES (2002)

Díaz, H., Reconocimiento de la productividad científica en Chile, Ingeniare, Revista Chilena de Ingeniería, 21(2), 170-171 (2013) 
El Aseguramiento de la Calidad en el contexto de la Reforma al Sistema: Algunos planteamientos generales (2015)

Embid, A. y F. Michavila, Hacia una nueva Universidad, Apuntes para un debate, Tecnos, Madrid, España (2001)

Espinoza, O. y L. González, Desarrollo de la formación de posgrado en Chile, 13 (5), 207-230 (2009)

Espinoza, O. y L. González, Estado actual del sistema de aseguramiento de la calidad y el régimen de acreditación en la educación superior en Chile, ISSN: 0185-2760, Revista de la educación superior, 162(41), 87-109 (2012)

Fondo Nacional de Desarrollo Científico y Tecnológico. Qué es FONDECYT (2017)

Grant, R., Prospering in Dynamically Competitive Environments. Organizational Capability as Knowledge Integration, Organization Science, $1^{\mathrm{a}}$ Ed., 375-387, School of Business, Washington, EUA (1996)

Innovación en la formación profesional, Revista Digital Buenas Prácticas, 2.0 (2013)

OCDE, El Aseguramiento de la Calidad en la Educación Superior en Chile (2013)

Oyarzun, R., Ciencia, revistas científicas y el Science Citation Index: o cómo volvernos locos a golpe de números. En Ciencia y Sociedad (2007)

Pedraja, L., C. Araneda, E. Rodríguez y J. Rodríguez, Calidad en la Formación Inicial Docente: Evidencia Empírica en las Universidades Chilenas, Formación Universitaria, 5(4), 15-26 (2012)

Pérez, M., Aseguramiento de la calidad de la educación superior en América Latina: ¿vamos por el camino correcto? Calidad en la Educación, (21), 271-285 (2018)

Restrepo, B., La investigación Formativa en los procesos de investigación asumidos en la universidad, uexternado.edu.co (2007)

Salas, R., La Calidad en el desarrollo profesional: avances y desafíos, Rev. Cubana Médica Superior, 14 (2), 136 -147 (2000)

Salazar, J., Diagnóstico preliminar sobre evaluación de la docencia universitaria. Una aproximación a la realidad en las universidades públicas y/o estatales de Chile, ISSN: 1989-0397, Revista Iberoamericana de Evaluación Educativa, 3(1), 68-83 (2008)

Sistema de Información Científica, Productividad Científica Nacional según formato de publicaciones WOS (2016)

Sistema de aseguramiento de la calidad de la Educación Superior: aspectos críticos y desafíos de mejoramiento, Centro de Políticas Públicas U.C., 45 (6) (2011)

Szilagyi, J. y T. Szecsi, Transforming teacher education in Hungary, Competencies for elementary teachers, Annual Theme, 327-331 (2011)

Teece, D., G. Pisano y A. Shuen, Dynamic Capabilities and Strategic Management, Strategic Management Journal, 18(7) 509-533 (1997)

Valera, J. y F. De la Gala, Análisis bibliométrico de la productividad científica, Mapfre Medicina Journal, 12, 157-167 (2001)

Vargas D., Medir la producción científica de los investigadores universitarios: la bibliometría y sus límites, Revista de la Educación Superior, XXXVI (142) (2007)

Zapata, G., Acreditación institucional en Chile: una opción emergente. Calidad en la Educación, (21), 141-154 (2018) 